\title{
Absence of adjuvant radiotherapy may be an additional criteria in diagnosing a branchiogenic squamous cell carcinoma: A case report
}

\author{
Alexandre Anesi ${ }^{1}$, Giuseppe Pollastri ${ }^{1}$, Vincenzo Bondì ${ }^{2}$, Sara Barberini ${ }^{2}$, Luigi Chiarini ${ }^{1}$ \\ ${ }^{1}$ Section of Cranio-Maxillo-Facial Surgery, Department of Head and Neck Surgery, University Hospital of Modena, Modena, Italy \\ ${ }^{2}$ Section of Oral and Maxillofacial Surgery, Department of Surgery, Azienda Ospedaliera Universitaria Integrata, Verona, Italy \\ Email: alexandre.anesi@unimore.it
}

Received 3 July 2011; revised 16 December 2011; accepted 5 January 2012

\begin{abstract}
Branchiogenic carcinoma is extremely rare. The majority of branchiogenic carcinomas are cystic metastases originating in the tonsils, and not true carcinomas arising in a branchial cleft cyst. Isolated cystic neck lesions necessitate a thorough search for a primary tumor, as with other occult primaries presenting with cervical metastases. As the existence of primary branchiogenic carcinoma is controversial, Martin and colleagues and then Khafif and coworkers established a series of widely accepted criteria to recognize this type of carcinoma. We report a case of a large cervical mass, consistent with a branchiogenic carcinoma of the second branchial arch. The patient underwent excision of the neck mass through a radical neck dissection. The cyst wall was found to have squamous cell carcinoma arising from the benign epithelium. Follow-up at 7 years revealed no evidence of recurrence. All diagnostic criteria for a true branchiogenic carcinoma were met in this case. The absence of adjuvant irradiation is a further confirmation that the mass is not a cystic node metastasis but a carcinoma arised from within a branchial cleft cyst.
\end{abstract}

Keywords: Branchial Cleft Cyst; Branchial Cyst; Branchiogenic Carcinoma; Malignant Branchioma; Cystic Cervical Lymph Node Metastasis

\section{INTRODUCTION}

A palpable neck mass is frequently seen by head and neck surgeons. Cystic masses of the neck include a wide range of congenital and acquired lesions. The majority of cystic lesions in children or young adults are congenital in origin, whereas in adults, inflammatory or neoplastic diseases constitute the more probable etiology. Developmental cysts include thyroglossal cysts, branchial cleft cysts and thymic cysts. Branchial cleft cysts are epithet- lial cysts that may arise from failure of obliteration and regression of the branchial cleft and pouch [1,2].

Depending on the series, branchial cleft and pouch anomalies account for about 30\% of all congenital head and neck lesions, with second branchial anomalies representing the overwhelming majority (95\%) [3].

Second branchial pouch may remain quiescent for many years and enlargement, squamous metaplasia and lymphoepithelial hyperplasia is a consequence of immunologic stimulation [3].

Branchiogenic carcinoma (BC) is a rare pathological entity in which the epithelial cells within the developmental cyst undergo a malignant transition [4,5]. The aetiology of malignant transition within a branchial cleft cyst is not exactly determinated. Proposed causative factors embrace head and neck irradiation and inflammatory reactions, but the aetiological relationships remain to be verified [3-5]. This carcinoma presents as a lateral neck swelling and usually arises in the fifth to sixth decades, more often in men than women. When diagnosis has been prolonged, later symptoms are caused by abscess formation or by the mass effect on the airway-digestive tract (dyspnea and/or dysphagia) [3,6].

The site of a second branchial cleft cyst, as well as the $\mathrm{BC}$, is embryologically defined, typically located in the lateral neck, superficial to the common carotid artery and internal jugular vein, posterior to the submandibular gland and along the medial and anterior margin of the sternocleidomastoid muscle (SCM) [3]. Several authors have expressed doubt about the existence of $\mathrm{BC}$ and concluded that it is a rare entity $[7,8]$. Histologically, it is difficult to distinguish branchial cleft carcinoma from cystic degeneration of squamous cell carcinoma metastatic from an occult primary in a cervical lymph node [9]. Martin and colleagues, and then Khafif and coworkers, established a series of widely accepted criteria to recognize this type of carcinoma $[6,10]$.

In this original report, a large cervical mass, consistent 
with a BC of the second branchial arch, is well described.

\section{CASE REPORT}

A 56-year-old Caucasian man without a specific history presented a painless mass on his right neck that had been there for 3 months. The patient was referred to our maxillofacial service under the suspicion of a suppurative lymphadenitis related to dental infection. However, on oral examination and dental panoramic radiograph no odontogenic infections were detected. Physical examination revealed a well-defined, movable, non-pulsatile and non-tender mass at the Level II-III zone of his right neck (Figure 1).

No other lymphadenopathy or thyroid mass was detected. He had no other complaints. He denied any previous cancer or radiation exposure. A bilateral tonsillectomy had been performed as a child.

On ultrasound, the cyst appeared ill-defined, heterogeneous, and thick-walled, containing internal septa. Fine needle aspiration cytology (FNAC) was not diagnostic for a infectious or neoplastic process. The aspirate yielded necrotic and inflammatory material with several granulocytes and rare cells with hyperchromatic eosinophil cytoplasm of uncertain significance, that merited

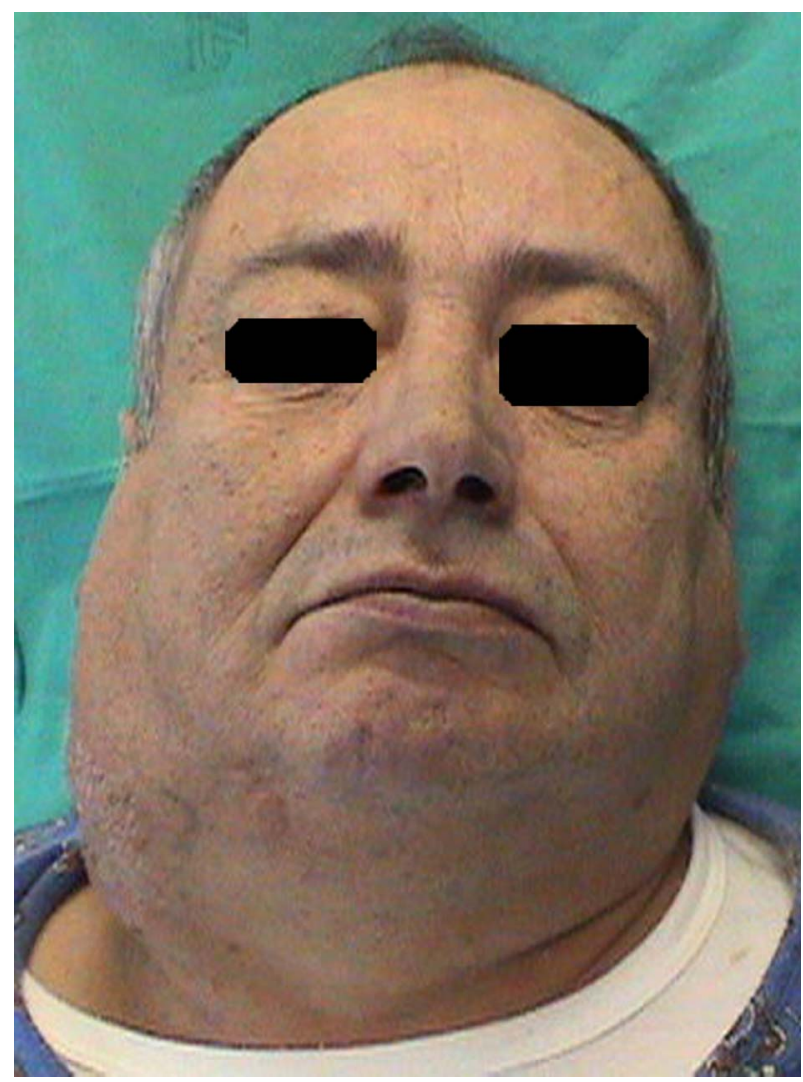

Figure 1. A frontal view of the patient with a large mass at the Level II-III zone of his right neck. further histologic investigation. MRI revealed an inhomogeneous cyst filled with liquid and containing septa, with medium intensity on T1-weighted images and higher intensity on T2-weighted images; a remarkable contrast enhancement was evident (Figures 2 and 3). It was located posterior to the submandibular gland, lateral to the great vessels, and anterior to the SCM in cranial

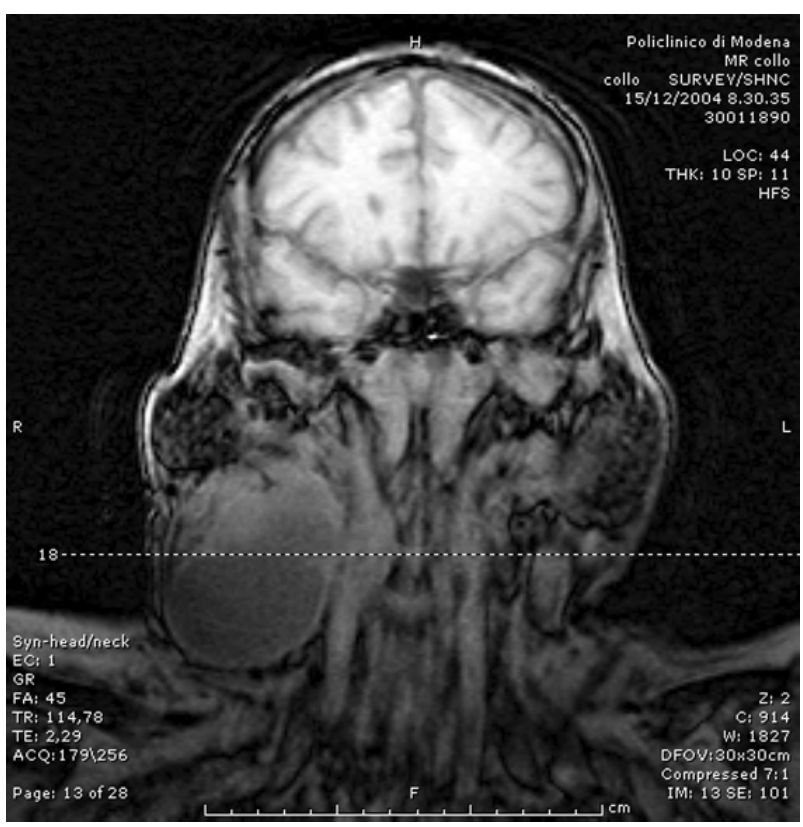

Figure 2. A frontal T1-weighted magnetic resonance image of the neck that shows the medium intensity of the lesion. The carotid space is displaced medially and anteriorly.

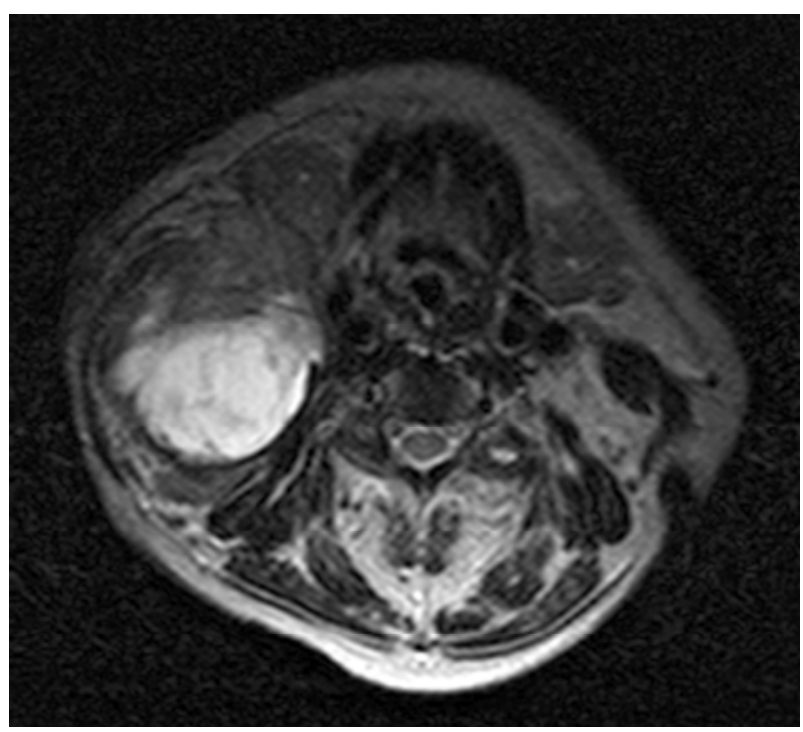

Figure 3. An axial T2-weighted magnetic resonance image of the neck. The image reveals a well-circumscribed 6-cm mass in the right anterior cervical space. Signal intensity is high due to the presence of intracystic fluid. The cyst is located posterior to the submandibular gland, and lateral to the great vessels, displacing the SCM posteriorly in cranial slices. 
slices; the caudal boundary of the mass was difficult to detect proximal to the clavicular insertion of the SCM. Under general anesthesia an incisional biopsy taken from the within the boundaries of the mass was performed. The intraoperative frozen section revealed atypical cellular nests "consistent with carcinoma" between fibrous tissue. Because the lesion was inseparable from the SCM, the patient underwent surgical removal of the cyst (6.7 $\mathrm{cm}$ in diameter) through a radical neck dissection (with sacrifice of the SCM, the jugular vein, and the spinal accessory nerve). The excised specimen showed an encapsulated cystic structure approximately 8 centimeters in diameter. The final histopathological report described an high grade epithelial malignancy with evidence of underlying lymphoid aggregates; peripheral fibrosis and intraluminal fibrotic septa delimited a cystic space full of hemorrhagic material. The tumor cells showed pleomorphism, evident atypical features and several mitoses. The surgical margins were negative for tumor. No other metastatic foci to other lymph nodes of the specimen were documented.

By immunohistochemistry, the tumoral cells showed strong staining for MNF-116 (mouse monoclonal antibody to cytokeratin 116), CK-7 (cytokeratin 7), and CK903 (cytokeratin 903), confirming the epithelial differentiation of the neoplastic tissue [11]. Leucocyte common antigens research was also negative. Mesenchymal origin of these cell was excluded since actin and vimentin antibodies were negative. S-100 staining was also negative; on the contrary neural crest cell neoplasm (melanoma, schwannoma, paraganglyoma) show this marker. Moreover, TTF1 (Thyroid Transcription Factor One) negative staining ruled out an origin from an occult thyroid carcinoma, confirming a primitive small cell tumor. Prognostic immunohistochemical factors showed strong staining for EGFR (Epidermal Growth Factor Receptor) and focal positivity for C-Kit (CD117).

The final histopathological diagnosis of the lesion was BC (Figure 4).

The patient received further clinical examination, including a thorough endoscopy of the upper airways and digestive tract with random biopsies, a chest X-ray, thyroid and abdominal ultrasonography and a bone scan. No other primary malignancy was found. The post-operative course was unremarkable. Post-operative irradiation was not administered since the patient subsequently refused the treatment. A follow-up at 7 years has revealed no evidence of recurrence.

\section{DISCUSSION}

As the existence of primary BC is controversial, in 1950, Martin et al. established four criteria to recognize and diagnose this type of carcinoma [6,10,11].

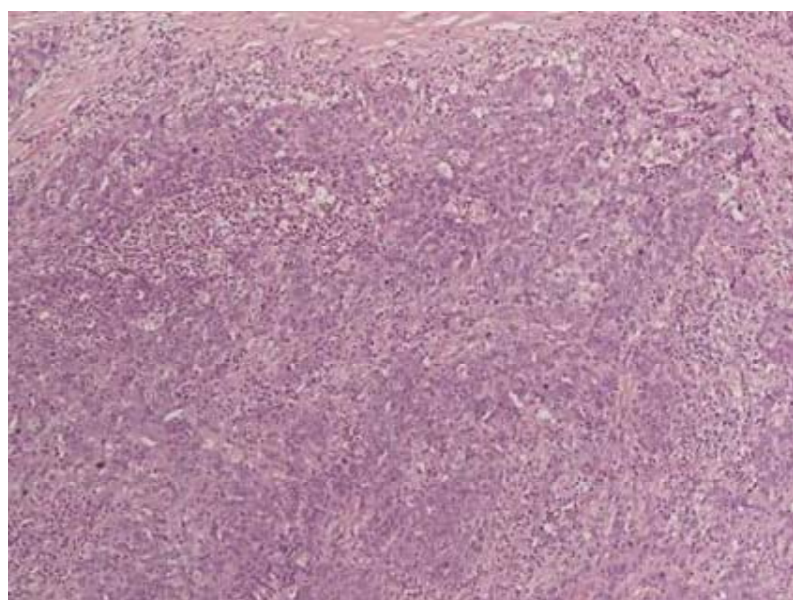

Figure 4. Photomicrograph showing an area of the branchial cystic wall with invasive and poorly-differentiated squamous cell carcinoma (hematoxylin-eosin stain).

1) The cervical tumor must have occurred somewhere along the line extending from a point just anterior to the tragus, downward along the anterior border of the sternocleidomastoid muscle to the clavicle $[6,10,11]$.

2) The histologic appearance of the growth must be consistent with an origin from tissue known to be present in the branchial vestiga $[6,10,11]$.

3) The patient must have survived and have been followed up for at least 5 years without the development of any other lesion that could possibly have been the primary tumor $[6,10,11]$.

4) The best criteria of all would be the histologic demonstration of cancer developing in the wall of an epithetlial cyst situated in the lateral aspect of the neck (a normal branchiogenic cyst) $[6,10,11]$.

In 1989, Khafif et al. proposed two additional criteria: 1) histological identification of transition from the normal squamous epithelium of the cyst to carcinoma and the 2) absence of any identifiable primary malignant tumor after exhaustive evaluation of the patient $[6,11]$.

Based on the criteria of these two groups, fewer than 40 cases of $\mathrm{BC}$ have been reported in the literature; many cystic cervical lesions labeled in the past as BC may now be considered as metastatic squamous cell cystic carcinoma originating in the oropharynx, nasopharynx, hypopharynx, larynx or oral cavity $[8,10]$. According to Thompson et al., a particular subtype of tonsillar squamous cell carcinoma in Waldeyer's ring is thought to form prominently cystic metastases in the upper jugular lymph nodes, difficult to diagnose and liable to be confused with BC $[4,8,9]$. Cystic cervical lymph node metastasis is strongly associated with HPV-related tonsillar squamous cancer [12]. Therefore, an isolated cystic neck lesion in an adult patient, particularly over 40 years, necessitates a thorough search for a primary tumor, before suspecting a branchial remnant $[6,10,12]$. 
Recommended diagnostic procedures include physical examination with evaluation by fiber-optic endoscopy of the upper airways and digestive tract (esophagus included), ultrasonography with FNAC, head and neck CT and/or MRI [11]. With ultrasound most uninfected second brachial cysts demonstrate the typical appearance of a cyst in that they are well-defined and anechoic. When complicated by previous infection/inflammation, they are usually ill-defined, heterogeneous, and thick-walled, containing internal debris and septa [1]. Such cysts mimic metastatic nodes from papillary carcinoma of thyroid or squamous cell carcinoma. In these cases, FNAC of any solid mural component is recommended to exclude a metastatic lymph node; cytologic diagnosis should always be confirmed by post-operative histologic examination [2,11]. With CT, a second branchial cleft cyst appears as a homogeneous mass with low attenuation and a thin, well-defined wall in the typical location. Conversely, infected cysts can be hyperattenuated with an ill-defined irregular rim, mimicking a metastatic node [1]. On MRI, the T2-weighted signal intensity is high due to the presence of intracystic fluid. The proteinaceous content of the cyst may sometimes cause it to appear hyperintense rather than isointense or hypointense on T1weighted series.

Since the onset of $\mathrm{BC}$ is rare, data on the management of true $\mathrm{BC}$ are scarce [8]. The natural course of $\mathrm{BC}$ is unpredictable because of the small number of cases that have been reported [9]. However, early lateral neck swelling with symptoms was reported by Khafif et al. with a more aggressive behavior of the lesion; these observations were not shared by Singh et al. [6,9]. Most authors would agree that the treatment for a presumed BC should be aggressive, because these tumors are highly invasive in their own right $[9,13]$. As suggested by Singh and colleagues, the therapeutic approach should be similar to that for an unknown head and neck primary malignancy, because most cases of presumed BC are finally found to be due to a cystic node metastasis [9]. Nevertheless, according to Zimmermann et al., the treatment of a cervical cyst with dysplasia or carcinoma in situ, without malignant infiltration, consists of complete surgical excision $[3,14]$. The surgical endoscopic procedure for resection of a non-malignant branchial cyst was recently described; specific surgical training in endoscopy is needed [15]. Endoscopic removal of the cyst is possible by two different techniques: an intracystic or extracystic endoscopic procedure. A proposed intracystic dissection in the BC lesion would be not correct in our opinion from an oncologic point of view, because of the risk of incomplete excision or spread of malignant cells in the operative field; we therefore urge extracystic endoscopic dissection rather than the intracystic technique for a non-infiltrated BC cyst.
When a infiltrative BC is suspected, a wide surgical excision is needed, preferably with ipsilateral neck dissection, conservatively if possible, followed by adjuvant radiotherapy and chemotherapy $[4,2,5,6,8,13]$. However, if the diagnosis of occult primary squamous cell carcinoma is in question after comprehensive examination (radiographic, endoscopic and bioptic), it is useful to emphasize that in upper jugular metastatic cystic squamous cell carcinoma, the surgical excision of the mass often removes all of the metastatic disease, since many of these lesions are solitary [8]. Adjuvant radio-therapy to one side of the neck and the oropharynx is suggested by several authors owing to the aggressive behavior of primary BC (possible metastasis to regional lymph nodes or a distant site), and because most cases of presumed BC are ultimately found to be a cystic metastasis from a tonsillar carcinoma $[8,12,13]$. In contrast, according to Singh et al., when all lymph nodes are negative for tumor in the neck specimen, adjuvant therapy might be avoided; the author reserves radiation therapy for when patients refuse further neck dissection, when metastatic lymph nodes are detected in the specimen and when the patient develops locoregional recurrence [9,13]. To resume, we have to consider some aspects of the treatment orientation process after surgical excision of a BC: 1) a long-term survival is reported in $\mathrm{BC}$ and cystic squamous cell carcinoma in the neck compared with the data observed for solid metastatic squamous cell carcinoma; 2) retropharyngeal node involvement is rare in tonsillar carcinoma; 3 ) the nasopharynx is not a very likely occult primary site compared to the oropharynx. So, in selected patients, irradiation might spare retropharyngeal nodes and the nasopharynx, reducing the post-irradiation effects [8].

In the English language literature, chemotherapy as adjuvant treatment in BC is reported in one paper by Katori et al. [5]. They described a weekly administration of carboplatin and daily oral tegafur-uracil (UFT) with concurrent radiotherapy [5]. Clinical follow-up with serial examination for the remainder of the patient's life is recommended due to an increased risk of recurrence, about 7\% with a latency time from 3 months up to 62 months $[2,10,11]$. Prognosis data in BC are scarce. However, we can argue a relatively long-term survival in patients with BC from studies on cystic metastatic squamous cell carcinoma, since these lesions have a more indolent growth than most squamous cell carcinomas. The 5-year survival for cystic metastatic carcinoma of the oropharynx is $77 \%$, enjoying a better prognosis than other head and neck squamous cell carcinomas [8].

According to Thompson and Heffner, our case of BC described here excluded some confounding factors common to previous studies reported in the literature: 1) tonsillectomy was performed in childhood, ruling out 
any occult primary; 2) random biopsies were performed in the upper airways and digestive tract; 3) a follow-up at 7 years revealed no later appearance of a misdiagnosed primary; 4) no other squamous cell carcinoma occurred in the upper airways and digestive tract; 5) no other regional or distant metastatic lymph nodes were present; 6) we did not perform any post-operative radiation therapy that might have eradicated the possible occult primary; 7) the $\mathrm{BC}$ occurred in the correct anatomic location for second branchial remnants [8].

All diagnostic criteria for a true BC were met in this case, although the existence of this malignancy remains controversial for some investigators $[7,8,11]$.

In our clinical case, the infiltration of the SCM was demonstrated by radiological and pathological findings; the infiltrative progression of a large BC cystic lesion has also been reported by Hong et al. and confirmed the aggressive behavior of this malignancy [13].

This report supports the axiom proposed by Sandiford and then by Thompson that postoperative irradiation of BC may treat a misdiagnosed primary $[8,16]$. Our patient refused the medical indication for adjuvant radiotherapy and he is now without evidence of disease in follow-up at 7 year. This peculiar fact excludes conversely a confounding factor in diagnosing the malignancy as a primary BC: the absence of irradiation on the ipsilateral neck is a further confirmation that the mass is not a cystic node metastasis but a carcinoma arised from within a branchial cleft cyst.

\section{REFERENCES}

[1] Wong, K.T., Lee, Y.Y., King, A.D. and Ahuja, A.T. (2008) Imaging of cystic or cyst-like neck masses. Clinical Radiology, 63, 613-22. doi:10.1016/j.crad.2007.12.007

[2] Girvigian, M.R., Rechdouni, A.K., Zeger, G.D., Segall, H., Rice, D.H. and Petrovich, Z. (2004) Squamous cell carcinoma arising in a second branchial cleft cyst. American Journal of Clinical Oncology, 27, 96-100. doi:10.1097/01.coc.0000047127.46594.C0

[3] Glosser, J.W., Pires, C.A. and Feinberg, S.E. (2003) Branchial cleft or cervical lymphoepithelial cysts: Etiology and management. The Journal of the American Dental Association, 134, 81-86.

[4] Park, S.S. and Karmody, C.S. (1992) The first branchial cleft carcinoma. Archives of Otolaryngology Head and Neck Surgery. 118, 969-971. doi:10.1001/archotol.1992.01880090085022

[5] Katori, H., Nozawa, A. and Tsukuda, M. (2005) Post- operative adjuvant chemoradiotherapy with carboplatin and 5-fluorouracil for primary branchiogenic carcinoma. Journal of Laryngology \& Otology, 119, 467-469. doi:10.1258/0022215054273241

[6] Khafif, R.A., Prichep, R. and Minkowitz, S. (1989) Primary branchiogenic carcinoma. Head Neck, 11, 153-163. doi:10.1002/hed.2880110209

[7] Micheau, C., Klijanienko, J., Luboinski, B. and Richard, J. (1990) So-called branchiogenic carcinoma is actually cystic metastases in the neck from a tonsillar primary. Laryngoscope, 100, 878-883. doi:10.1288/00005537-199008000-00015

[8] Thompson. L.D. and Heffner, D.K. (1998) The clinical importance of cystic squamous cell carcinomas in the neck: A study of 136 cases. Cancer, 82, 944-956. doi:10.1002/(SICI)1097-0142(19980301)82:5<944::AIDCNCR21>3.0.CO;2-\#

[9] Singh, B., Balwally, A.N., Sundaram, K., Har-El, G. and Krgin, B. (1998) Branchial cleft cyst carcinoma: Myth or reality? Annals of Otology, Rhinology, and Laryngology, 107, 519-24.

[10] Martin, H., Morfit, H.M. and Ehrlich, H. (1950) The case for branchiogenic cancer (malignant branchioma). Annals of Surgery, 132, 867-887. doi:10.1097/00000658-195011000-00002

[11] Jereczek-Fossa, B.A., Casadio, C., Jassem, J., Luzzatto, F., Viale, G., Bruschini, R., et al. (2005) Branchiogenic carcinoma-Conceptual or true clinico-pathological entity? Cancer Treatment Reviews, 31, 106-114. doi:10.1016/j.ctrv.2004.12.007

[12] Goldenberg, D., Begum, S., Westra, W.H., Khan, Z., Sciubba, J., Pai, S.I., Califano, J.A., Tufano, R.P. and Koch, W.M. (2008) Cystic lymph node metastasis in patients with head and neck cancer: An HPV-associated phenomenon. Head Neck, 30, 898-903. doi:10.1002/hed.20796

[13] Hong, K.H., Moon, W.S. and Chung, G.H. (1999) Radiological appearance of primary branchial cleft cyst carcinoma. Journal of Laryngology \& Otology, 113, 1031-1033. doi:10.1017/S0022215100145918

[14] Zimmermann, C.E., von Domarus, H. and Moubayed, P. (2002) Carcinoma in situ in a lateral cervical cyst. Head Neck, 24, 965-969. doi:10.1002/hed.10118

[15] Kitano, H., Fujimura, M., Hirano, M., Sato, I., Kataoka, H., Kinoshita, T., et al. (2000) Endoscopic surgery for lateral cervical cysts. A report of three cases. Surgical Endoscopy, 14, 1086.

[16] Sandiford, J.A., Chun, B.K. and Potter, J.F. (1987) Branchiogenic carcinoma. Journal of the Royal College of Surgeons of Edinburgh, 32, 148-151. 